\title{
Preparation of cationic polyacrylamide by aqueous two-phase polymerization
}

\author{
J. Xu, W. P. Zhao, C. X. Wang, Y. M. Wu* \\ College of Chemical Engineering, Qingdao University of Science and Technology, Qingdao 266042, P. R. China
}

Received 7 January 2010; accepted in revised form 4 March 2010

\begin{abstract}
Cationic polyacrylamide (CPAM) was synthesized by aqueous two-phase polymerization technique using acrylamide (AM) and dimethylaminoethyl methacrylate methyl chloride (DMC) as raw materials, aqueous polyethylene glycol 20000 (PEG 20000) solution as dispersant, 2,2'-azobis(2-amidinopropane) dihydrochloride (V-50) as initiator and poly(dimethylaminoethyl methacrylate methyl chloride) (PDMC) as stabilizer. The polymer was characterized by infrared (IR) spectroscopy, ${ }^{1} \mathrm{H}-\mathrm{NMR}$ spectrum and transmission electron microscopy (TEM). The copolymer composition was analyzed. The effect of monomers concentration, PEG 20000 concentration and stabilizer concentration on copolymer were investigated, respectively. The optimum reaction conditions for obtaining a stable CPAM aqueous two-phase system were monomers concentration 8 15\%, PEG 20000 concentration 15 25\%, and PDMC concentration $0.5 \sim 1.5 \%$. Finally, the formation process of copolymer particles was investigated by optical microscope.
\end{abstract}

Keywords: polymer synthesis, aqueous two-phase polymerization, cationic polyacrylamide, dimethylaminoethyl methacrylate methyl chloride, polyethylene glycol 20000

\section{Introduction}

Cationic polyacrylamides (CPAM) are a kind of important cationic polyelectrolyte and extensively used as flocculants for liquid/solid separation, retention and drainage aids in papermaking, flotation aids and demulsifiers for oil/water clarification, as soil improvers and drainage aids, etc. [1-3]. Various types of methods for producing CPAM have been developed, such as homogeneous aqueous solution polymerization, inverse emulsion polymerization, inverse suspension polymerization and dispersion polymerization, and so on $[4,5]$.

For homogeneous aqueous solution polymerization, the reaction heat was difficult to remove because the material became gel which can not flow. For the sake of transportation and dissolution easily, CPAM gel was used to be handled as dry powder instead of aqueous solution. However, these polymers in dry-powder form not only required consumption of energy in drying process at elevated temperature but also were subjected to degradation through shear or three-dimensional polymer crosslinking. For the inverse emulsion polymerization and inverse suspension polymerization, some surfactants and organic solvents were used in the course of syntheses, definitely resulting in the problems of environmental pollution and recycling [4-6]. Recently, aqueous dispersion polymerization was used to prepare cationic polymerization, which could be carried out in aqueous tert-butyl alcohol media [7-9] or in the aqueous inorganic salt media $[6,10,11]$. Especially, the dispersion polymerization in aqueous solution of ammonium sulfate attracted considerable attention because of its organic-free solvent. However, the use of an amount of inorganic salt would restrict its development and the extensive applications. For example, if the cationic polymerization was used as retention aid in paper-

*Corresponding author, e-mail: wuyumin001@126.com

(c) BME-PT 
making industry, excessive ammonium sulfate likely made paper become brittle.

The concept of aqueous two-phase system was presented in the 1950s, and the technique of aqueous two-phase separation was mature in the field of biotechnology for the separation and purification of valuable biomolecules [12-15]. Aqueous twophase polymerization may be defined as a type of aqueous dispersion polymerization. In aqueous two-phase polymerization, the inorganic salt used in the aqueous dispersion polymerization was replaced by a water-soluble polymer. Polymerization reaction started from a homogeneous mixture of the water-soluble monomers, initiator, another water-soluble polymer and a stabilizer. The solvent was selected on the basis of solubility of monomers and stabilizer and insolubility of the polymer [16]. Polymerization was initiated in the aqueous solution by an initiator that was soluble in both the solvent and monomer. It may be used to prepare the water-soluble (co)polymers dispersion with good stability and high molecular weight which could be directly diluted to use. Aqueous two-phase polymerization overcame the problems of heat release of aqueous solution polymerization and avoided the environmental pollution and recycling questions of organic solvents in inverse suspension polymerization, water-in-oil inverse emulsion polymerization and precipitation polymerization [4-6]. Aqueous two-phase polymerization was an attractive alternative to other polymerization processes. However, to our knowledge, there were a few works on the preparation of water-soluble polymers by aqueous two-phase polymerization technique. Only Shan et al. successfully prepared the nonionic polyacrylamide by aqueous two-phase polymerization in the presence of PEG by using ammonium persulfate as the initiator and sodium dodecylsulfate or polyoxyethylene(20) sorbitan monolaurate as the emulsifier [17]. The stability of dispersion system with surfactants was weak and its viscosity was large. Until now, there are few works on synthesis of cationic polyacrylamide via aqueous two-phase polymerization without emulsifier.

Based on the theory above mentioned, the aqueous two-phase copolymerization of acylamide (AM) and dimethylaminoethyl methacrylate methyl chloride(DMC) was carried out in aqueous polyethylene glycol 20000 (PEG 20000) solution with 2,2'azobis(2-amidinopropane) dihydrochloride (V-50) as initiator. And the poly(dimethylaminoethyl methacrylate methyl chloride) (PDMC), which acted as polymeric stabilizer, was first applied to lower dispersion viscosity and improve the stability of the synthetic system. Comparing to the system without PDMC as the polymeric stabilizer, some different results were obtained.

\section{Experimentals}

\subsection{Materials}

Acrylamide (AM, A. R. Grade) was purchased from Dia-Nitrix Co. Ltd. (Japan) and was used as received. Dimethylaminoethyl methacrylate methyl chloride (DMC, 78\% aqueous solution, industrial grade) was from Mitsubishi Gas Chemical Company, INC and used without further purification. Polyethylene glycol 20000 (PEG) and 2,2'-azobis(2-amidino-propane) dihydrochloride (V-50; WAKO Pure Chemical Industries Ltd) were of analytical grade, and used as received. Poly(dimethylaminoethyl methacrylate methyl chloride) (PDMC), as $20 \%$ aqueous solution, was prepared from DMC in an aqueous solution using V-50 as initiator at $60^{\circ} \mathrm{C}$. The molecular weight of PDMC was $3.9 \cdot 10^{5} \mathrm{~g} \cdot \mathrm{mol}^{-1}$. Deionized water was used throughout this work. Other reagents were A. R. grade and used directly.

\subsection{Preparation of CPAM by aqueous two-phase copolymerization}

Varying quantities of ingredients containing monomers (AM and DMC), PEG, PDMC and deionized water were added to a $250 \mathrm{ml}$ glass reactor fitted with a stirrer, a reflux condenser, a thermometer and a nitrogen inlet tube. After bubbling purified nitrogen through the solution for $30 \mathrm{~min}$ utes and controlling system temperature at $65^{\circ} \mathrm{C}$ by a water bath, the monomers were initiated by addition of V-50 solution into the reaction system under invariable stirring. The reactions were proceeded for $8 \sim 10 \mathrm{~h}$ to get a high overall conversion of monomers.

\subsection{Characterization}

The generated sample was precipitated repeatedly in a large quantity of acetone to remove the unreacted monomers and the PEG 20000. Then, the pre- 
cipitated CPAM copolymer was dried to a constant weight at $45^{\circ} \mathrm{C}$ under vacuum. The dried sample was used to determine the intrinsic viscosity in $1.0 \mathrm{~mol} \cdot \mathrm{l}^{-1} \mathrm{NaCl}$ at $30^{\circ} \mathrm{C}$ using an Ubbelohde viscometer [18]. The following Equation (1) was used to calculate the molecular weight of copolymer [10]:

$[\eta]=3.73 \cdot 10^{-4}\left[M_{w}\right]^{0.66}$

The viscosity of the aqueous two-phase system was measured by Brookfiled DV-II+Viscometer at the room temperature. The average particle size and particle size distribution (PDI) was measured by a laser particle size analyzer (Zeta-Sizer 3000, Malvern, UK) after ultrasonic dispersion for 20 minutes in acetone. The particle morphology was observed by optical microscope and transmission electron microscopy (JEM-1200EX; JEOL, Japan). FT-IR spectra were recorded on a spectrophotometer (AVATAR 360, Nicolet, USA) using $\mathrm{KBr}$ pellets. The ${ }^{1} \mathrm{H}-\mathrm{NMR}$ spectrum of AM/DMC copolymer was obtained in $\mathrm{D}_{2} \mathrm{O}$ with a Bruker $(500 \mathrm{MHz}){ }^{1} \mathrm{H}-\mathrm{NMR}$ spectrometer (Bruker Corporation, Bremen, Germany).

The overall monomer conversion could be obtained by determining the residual contents of $\mathrm{AM}$ and DMC with bromating method [6]. About $4.0 \mathrm{~g}$ of copolymer dispersion was weighed out in a weighing bottle, and then $100 \mathrm{ml}$ of deionized water was added. After stirring adequately, the sample solution could be obtained. Excessive $\mathrm{KBrO}_{3}-\mathrm{KBr}$ was added into the sample solution. In the presence of $\mathrm{H}^{+}, \mathrm{KBrO}_{3}$ reacted with $\mathrm{KBr}$ to produce $\mathrm{Br}_{2}$, and then $\mathrm{Br}_{2}$ could react with residual $\mathrm{AM}$ and DMC in the sample solution for addition reaction. Excessive KI was used to react with the residual $\mathrm{Br}_{2}$, and $\mathrm{I}_{2}$ was formed from the reaction mixture. By titrating $\mathrm{I}_{2}$ with the standard solution of $\mathrm{Na}_{2} \mathrm{~S}_{2} \mathrm{O}_{3}$ and calculating the consumption of $\mathrm{Na}_{2} \mathrm{~S}_{2} \mathrm{O}_{3}$, the total residual contents of AM and DMC could be determined by means of the interrelationship of these ingredients.

\section{Results and discussion}

The concentrations of initiator and stabilizer were weight percent based on total monomers, and the content of monomers and dispersant were weight percent based on total raw reaction materials. The amount of each ingredient was kept constant in all experiments, except as indicated. $X_{\mathrm{DMC}}$ indicated the mole percentage of DMC based on the initial monomers composition.

\subsection{Effect of monomers concentration}

Figure 1 shows the monomers conversion versus time curves with different monomers concentrations. It was shown that the initial polymerization rate and the overall conversion increased with the increase of monomers concentration. As shown in Figure 1, when the monomers concentration was $8 \%$, the overall monomers conversion was only $12 \%$ corresponding to the time at 30 . However, when the monomers concentration was $20 \%$, the overall conversion was $70 \%$ corresponding to the time at 30. This indicated that the lower the monomers concentration was, the stronger the shielding effect of aqueous PEG 20000 solution on monomers radicals was. So, the initiation efficiency of initiator decreased which resulted in decreasing of both the polymerization rate and the overall conversion. When the monomer concentration was higher than $20 \%$, the dispersion was so unstable that coagulation occurred. As the monomers concentration increased, the initial polymerization rate increased and much more oligomers were formed in the aqueous PEG solution. Yet the phase separation effect of PEG and stabilization of PDMC were weakened. So, the stability of copolymer particles in the medium decreased and copolymer particles coagulated gradually.

The effects of the monomers concentration on the relative molecular weight, the average particle size

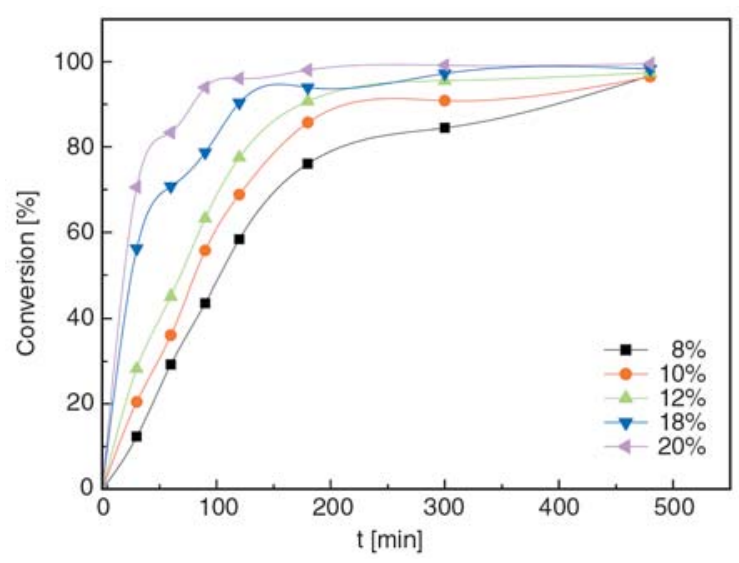

Figure 1. Conversion versus time curves with different monomers concentration. Polymerization conditions: $X_{\mathrm{DMC}} 10 \%$, PEG 25\%, V-50 0.009\%, PDMC $1 \%$ and $T 65^{\circ} \mathrm{C}$. 
Table 1. Effect of monomers concentration on aqueous two-phase system

\begin{tabular}{|c|c|c|c|c|}
\hline $\begin{array}{c}\text { Monomers } \\
{[\%]}\end{array}$ & $\begin{array}{c}M_{W} \\
{\left[10^{-6} \cdot \mathrm{g} \cdot \mathrm{mol}^{-1}\right]}\end{array}$ & $\begin{array}{l}\text { P.D. }{ }^{a} \\
{[\mathrm{~nm}]}\end{array}$ & $\begin{array}{c}\eta \\
{[\mathrm{mPa} \cdot \mathrm{s}]}\end{array}$ & Characteristics \\
\hline 8 & 1.15 & 716.8 & 4567 & Gray-white, good fluidity, and low viscosity dispersion \\
\hline 10 & 1.32 & 759.7 & 5065 & Gray-white, good fluidity, and low viscosity dispersion \\
\hline 12 & 1.45 & 839.6 & 5723 & Milky-white, good fluidity, and low viscosity dispersion \\
\hline 15 & 1.69 & 956.7 & 5998 & Milky-white, good fluidity, and low viscosity dispersion \\
\hline 20 & 1.89 & 1168.5 & 7596 & Milky-white, good fluidity, and high viscosity dispersion \\
\hline
\end{tabular}

aP.D. average of particle diameter;

Polymerization conditions: $X_{\mathrm{DMC}} 10 \%$, PEG $25 \%, \mathrm{~V}-500.009 \%$, PDMC $1 \%$ and $T 65^{\circ} \mathrm{C}$

and the apparent viscosity of dispersion were shown in Table 1. It was seen that the relative molecular weight, the average particle size and the apparent viscosity of dispersion all increased with the increase of monomers concentration, which conformed to the rules of typical free radical polymerization [19]. When the monomers concentration was up to $20 \%$, the viscosity of dispersion drastically increased on such condition, the dispersion was so unstable that coagulation occurred.

\subsection{Effect of PEG 20000 content}

High molecular weight PEG 20000, which had a tendency to interact with water through hydrogen bonding, was chosen as the phase separation agent because it formed aqueous two-phase system at lower concentrations and hence of lower viscosities [12]. To investigate the effect of the PEG 20000 content on the aqueous two-phase copolymerization system, five different PEG 20000 content (i.e. $5,10,15,20$, and $25 \%$ ) were examined. Figure 2 shows the relationship between the conversion and time in five different PEG 20000 concentrations. It was clear that high PEG 20000 content retarded the initial copolymerization rate and decreased the overall monomers conversion, which was contrary to the work that Shan and Cao reported [17]. It was due to that the following: as the PEG 20000 content increased, the viscosity of the continuous phase increased correspondingly, which prevented monomer radicals to be freely transferred between

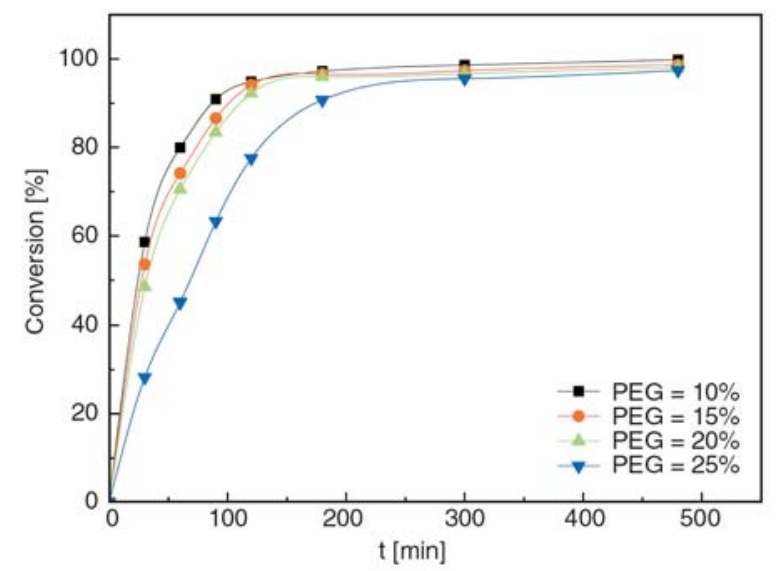

Figure 2. Conversion versus time curves with different PEG 20000 content. Polymerization conditions: total monomer $8 \%, X_{\text {DMC }} 10 \%$, PEG $25 \%, \mathrm{~V}-50$ $0.009 \%$, PDMC $1 \%$ and $T 65^{\circ} \mathrm{C}$.

mature particles and continuous phase. So the growth of copolymer particles became slower. At the same time, the shielding effect to free radicals was improved by PEG 20000. Thus, the initial copolymerization rate and the final overall conversion were both reduced.

As shown in Table 2, the molecular weight of copolymers and the apparent viscosity of the system lowered with increasing the PEG content. The average particle size and PDI also decreased. The reason was that increasing the PEG content resulted in the critical chain length to phase-out shorter. Therefore, the viscosity of the continuous phase decreased and the particle size as well as particle size distribution became smaller [11]. Meanwhile, the PEG 20000 had active $\alpha$-hydrogen atoms and

Table 2. Effect of PEG 20000 concentration on aqueous two-phase polymerization

\begin{tabular}{|c|c|c|c|c|c|}
\hline $\begin{array}{c}\text { WPEG } \\
{[\%]}\end{array}$ & $\begin{array}{c}M_{w} \\
{\left[10^{-6} \cdot \mathrm{g}^{\prime} \mathrm{mol}^{-1}\right]}\end{array}$ & $\begin{array}{c}\eta \\
{[\mathrm{mPa} \cdot \mathrm{s}]}\end{array}$ & $\begin{array}{l}\text { P.D. } \\
{[\mathrm{nm}]}\end{array}$ & PDI & Characteristics \\
\hline 5 & 1.04 & 10450 & 969.6 & 0.519 & High viscosity, large particles, and phase separation \\
\hline 10 & 0.99 & 6486 & 836.3 & 0.497 & High viscosity, large particles, and phase separation \\
\hline 15 & 0.92 & 6070 & 814.9 & 0.480 & Milky-white, good fluidity, and low viscosity dispersion \\
\hline 20 & 0.91 & 4946 & 801.5 & 0.430 & Milky-white, good fluidity, and low viscosity dispersion \\
\hline 25 & 0.84 & 4736 & 716.8 & 0.369 & Milky-white, good fluidity, and low viscosity dispersion \\
\hline
\end{tabular}

Polymerization conditions: total monomer $8 \%, X_{\mathrm{DMC}} 10 \%$, V-50 0.009\%, PDMC $1 \%$ and $T 65^{\circ} \mathrm{C}$ 

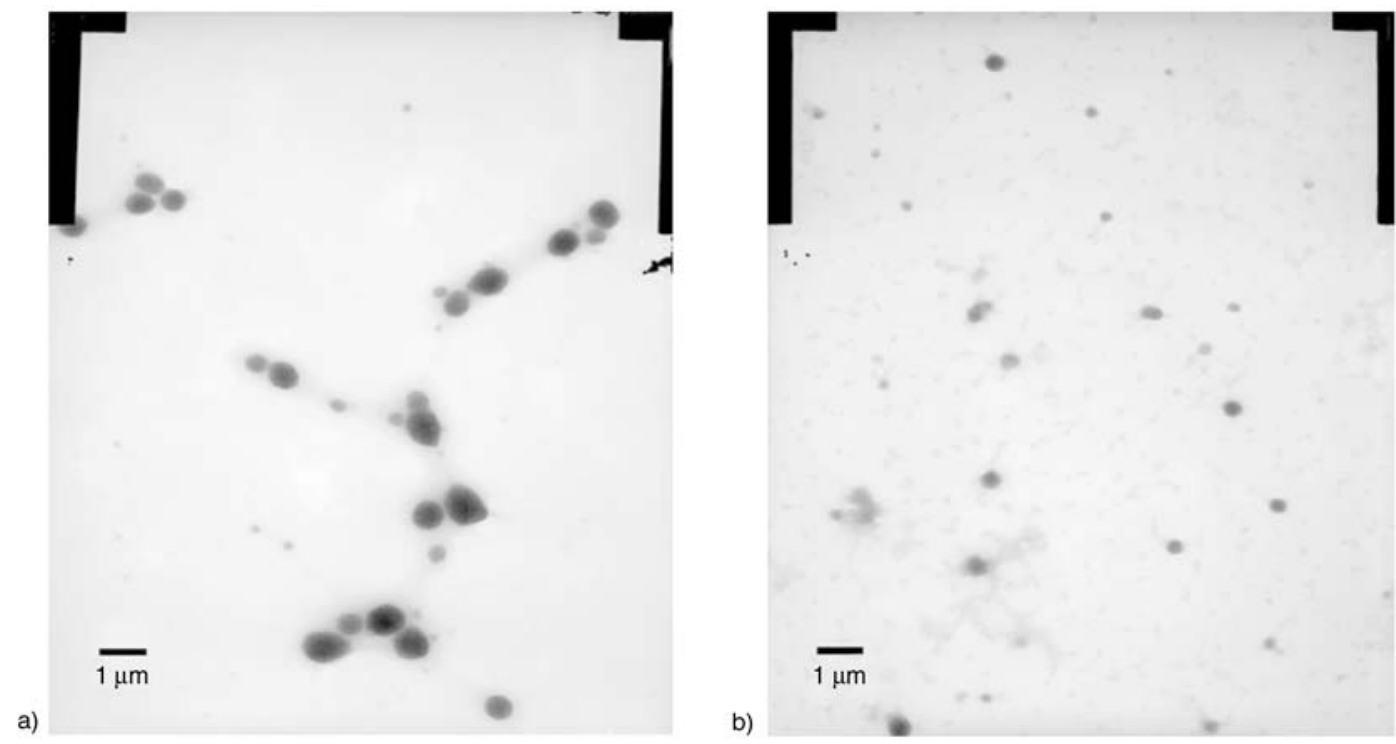

Figure 3. TEM photos of CPAM particles prepared in aqueous two-phase polymerization system with different PEG 20000 content: (a) $15 \%$, (b) $25 \%$

acted as chain transfer agent. Thus, the relative molecular weight decreased with PEG 20000 increasing. In addition, it was observed that the unstable dispersions were obtained when the PEG 20000 concentration was less than $10 \%$. Under such conditions, the polymerization of most monomers occurred in the continuous phase because the ability of phase separation decreased.

Figure 3 shows the TEM photos of CPAM particles in aqueous two-phase polymerization system with different PEG 20000 contents. It revealed that the coalescence between polymer particles took place and the particle size was larger when the PEG 20000 content was $15 \%$, to a certain extent. But, when the PEG content was $25 \%$, no coalescence was observed and the particle size of polymer particles was smaller.

\subsection{Effect of stabilizer concentration}

In the system, a water-soluble homopolymer PDMC was used as a steric stabilizer. Figure 4 shows that there was a little effect of PDMC concentration on the initial polymerization rate and the

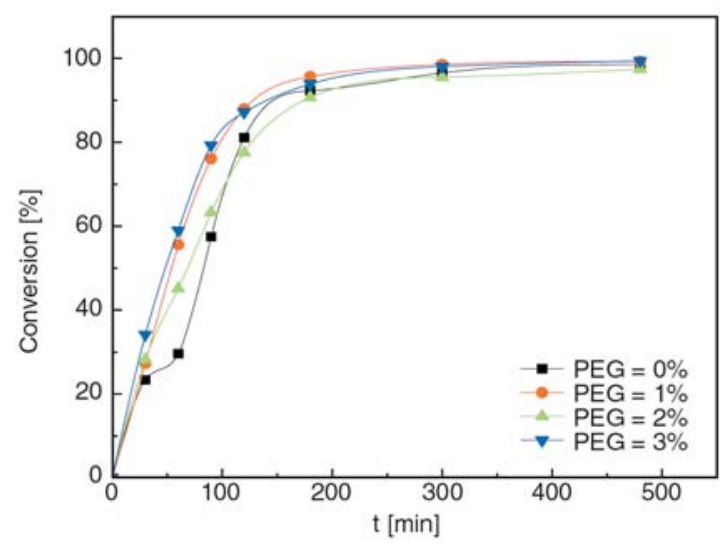

Figure 4. Conversion versus polymerization time curves with different PDMC concentrations. Polymerization conditions: Total monomers $8 \%, X_{\mathrm{DMC}} 12 \%$, $\mathrm{V}-500.009 \%$, PEG $25 \%$ and $T 65^{\circ} \mathrm{C}$.

overall conversion. The reason was that at the earlier stage of polymerization, monomers polymerization mainly took place in the dispersion media, adding a small amount of PDMC into the continuous phase had a little effect on the initiator.

From the results of Table 3, it was revealed that the relative molecular weight increased with PDMC concentration, which was consistent with some works reported $[1,2]$. With the increase of the sta-

Table 3. Effect of stabilizer PDMC concentration on CPAM aqueous two-phase system

\begin{tabular}{|c|c|c|c|c|}
\hline $\begin{array}{c}\text { PDMC } \\
{[\%]}\end{array}$ & $\begin{array}{c}M_{w} 10^{-6} \\
{\left[10^{-6} \cdot \mathrm{g}^{\prime} \mathrm{mol}^{-1}\right]}\end{array}$ & $\begin{array}{c}\eta \\
{[\mathrm{mPa} \cdot \mathrm{s}]}\end{array}$ & $\begin{array}{l}\text { P.D. } \\
{[\mathrm{nm}]}\end{array}$ & Characteristics \\
\hline 0.0 & 0.65 & 3280 & 773.1 & Phase separation after 3 months \\
\hline 0.5 & 0.76 & 3217 & 763.2 & Milky-white, smooth, still stable after 6 months \\
\hline 1.0 & 0.82 & 5065 & 758.5 & Milky-white, smooth, still stable after 6 months \\
\hline 1.5 & 0.94 & 5156 & 843.6 & Milky-white, smooth, still stable after 6 months \\
\hline 2.0 & 0.95 & 7615 & 931.5 & Phase separation after 7 days \\
\hline
\end{tabular}


bilizer concentration, a greater number of smaller particles were stabilized for the high stability. The smaller particle can effectively capture growing oligomeric radicals initiated in continuous phase. Solid-phase polymerization occurred to yield the polymer of high molecular weight due to the gel effect. Meanwhile, both the apparent viscosity of dispersion and the average particle size decreased first and then increased, which was contrary to other works related to aqueous dispersion polymerization $[1,8]$. The difference may be due to the absence of inorganic salt. Increasing the stabilizer concentration, the average particle size decreased. When PDMC concentration was $1 \%$, the particles with the smallest particle size were obtained. How-

a)
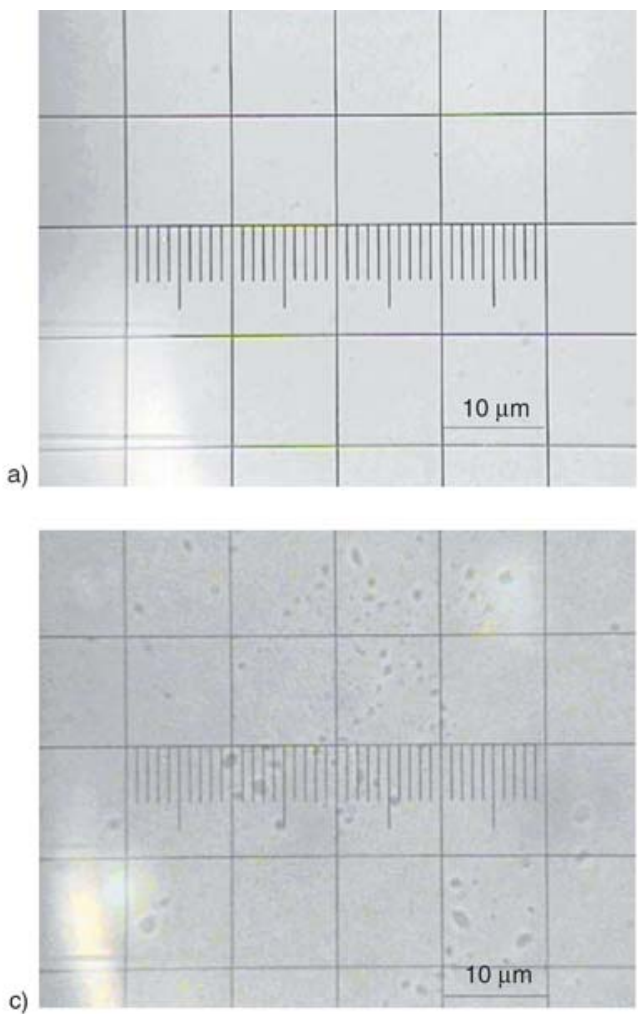

ever, beyond this point, the average particle size increased with PDMC concentration. Firstly, when PDMC concentration was below $1 \%$, PDMC was mainly acted as stabilizer and protect particles from coalescence. As the PDMC concentration increased from 0 to $0.5 \%$, both the average particle size and the viscosity of dispersion decreased. However, when the PDMC concentration was too high $(1.0 \%$ and above), the viscosity of system and the average particle size increased, but the stability of dispersion became weak. This was probably due to that extra stabilizer molecules did not participate in stabilization, but they had bridging role between the particles, inducing agglomeration. So the apparent viscosity and the particle size both increased again.

b)

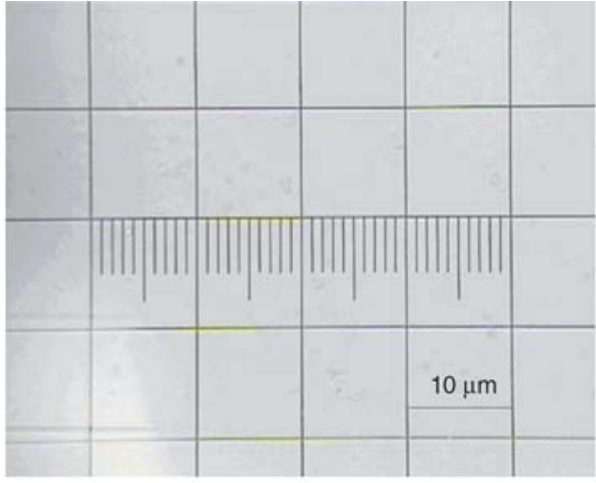

d)

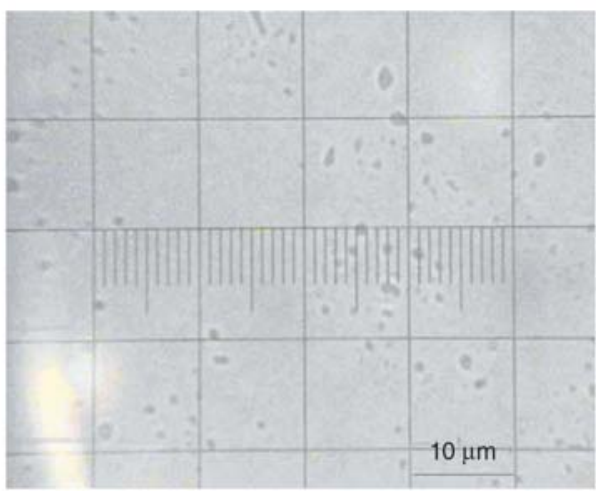

e)

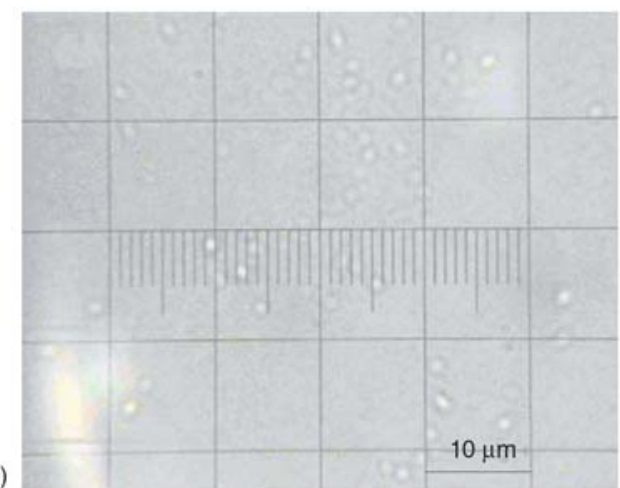

Figure 5. Microstructure of dispersed droplets in the aqueous two-phase polymerization system 


\subsection{Formation of dispersed droplets in aqueous two-phase polymerization}

Figure 5 showed the microstructure of the dispersed droplets in the aqueous two-phase polymerization system. The system was homogeneous before the polymerization (Figure 5a). As soon as the polymerization started, the primary free radicals grew in the dispersion media by addition of initiator until they reached their critical chain length. Then, they precipitated from the dispersion media to form nuclei. These nuclei with higher surface energy were unstable and quickly aggregated each other to form mature particles (Figure 5b). As the polymerization proceeded, the number of the mature particles in media became larger. At the same time, the mature particles grew by capturing the monomers, oligomeric radicals and nuclei in the dispersion media (Figure 5c). Because the particles consumed an amount of oligomeric radicals and nuclei, the rate of formation of particle reduced. Particles continually captured monomers from the dispersion media to form larger particles (Figure 5d). Until the monomers were nearly used up, the volume of particles did not change any longer (Figure 5e).

\subsection{Characteristics of CPAM aqueous two-phase}

Figure 6 shows the FTIR spectra for the final copolymer. The peaks at $3300 \sim 3500 \mathrm{~cm}^{-1}$ were due to amine group, whereas the peak at 1659 and $952 \mathrm{~cm}^{-1}$ was due to amide group and quaternary

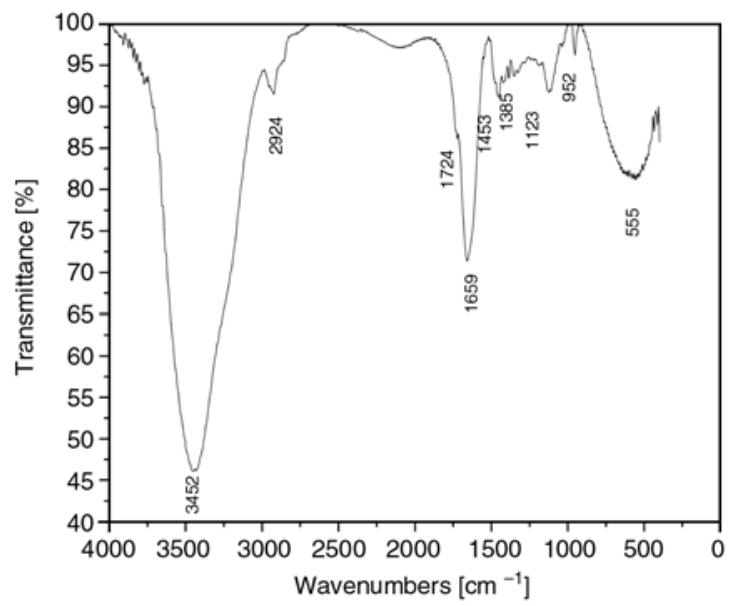

Figure 6. IR spectra of $\mathrm{P}(\mathrm{AM} / \mathrm{DMC})$

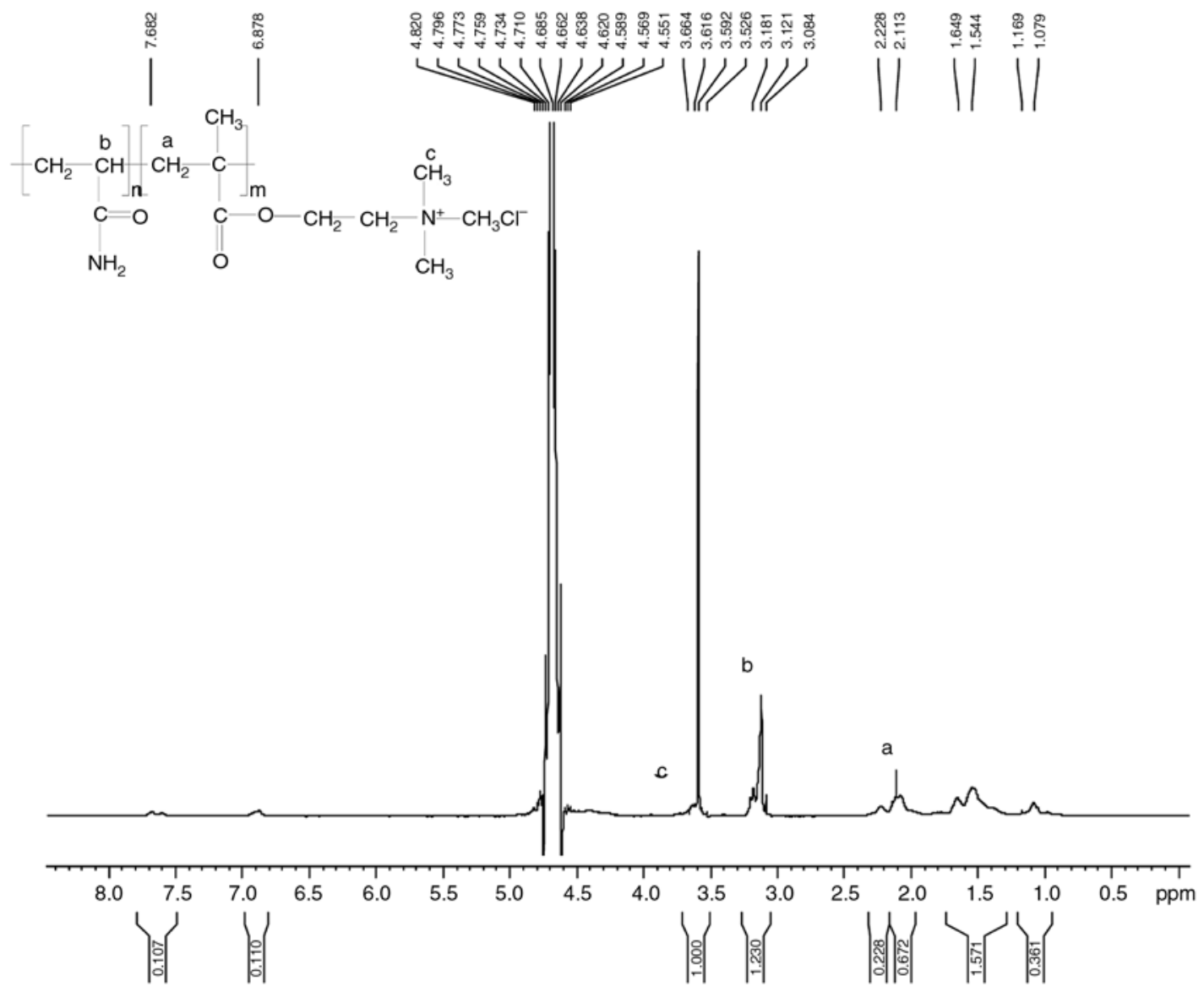

Figure 7. ${ }^{1} \mathrm{H}-\mathrm{NMR}$ spectra of AM with DMC copolymer $\left(X_{\mathrm{DMC}}=10 \%\right)$ 
ammonium. The peaks at 2924, 1724 and $1453 \mathrm{~cm}^{-1}$ were assigned to $\mathrm{v}-\mathrm{CH}_{3}, \mathrm{v}-\mathrm{O}-\mathrm{CO}-$ and $\delta-\mathrm{CH}_{2}-\mathrm{N}^{+}\left(\mathrm{CH}_{3}\right)_{3}$, respectively. It was believed that the final formed copolymer was $\mathrm{P}(\mathrm{AM} / \mathrm{DMC})$. Figure 7 represents ${ }^{1} \mathrm{H}-\mathrm{NMR}$ spectra of the $\mathrm{P}(\mathrm{AM} /$ $\mathrm{DMC}$ ). Each proton was readily distinguished from the resonance peaks of the copolymer. According to the integral area of the resonance peaks of $\mathrm{b}$ and c proton $\left(-\mathrm{CH}\right.$ of $\mathrm{AM}$ and $-\mathrm{CH}_{3}$ of $\left.\mathrm{DMC}\right)$, the approximate composition of the copolymer could be calculated. The molar ratio of AM to DMC in the copolymer is about $88 / 12$, which was near to the ratio in the feed, 90/10. The copolymer composition determined via ${ }^{1} \mathrm{H}-\mathrm{NMR}$ spectroscopy was in good agreement with the molar feed compositions and typically deviated no more than $2 \mathrm{~mol} \%$ from the feed values.

\section{Conclusions}

In this work, cationic polyacrylamide (CPAM) was synthesized by aqueous two-phase polymerization technology. The initial polymerization rate, the overall conversion of monomers, the relative molecular weight, the average particle size, and the apparent viscosity of dispersion all increased with monomers concentration, contrary to PEG 20000 concentration. There was a little effect on initial polymerization rate and the overall conversion with PDMC concentration. As the PDMC concentration ranged from 0 to $0.5 \%$, the relative molecular weight increased and both particle size and the viscosity of dispersion decreased. However, the relative molecular weight, particle size and the viscosity of dispersion both increased when the PDMC concentration was higher than $1 \%$. The stable conditions for CPAM aqueous two-phase system were as follows: $8 \sim 15 \%$ for monomers concentrations, 15 25\% for PEG 20000, 0.5 1.5\% for PDMC. Meanwhile, the formation process was investigated by optical microscope.

Finally, it should be noted that keeping lower viscosity and avoiding the Wessenberg effect (when the system viscosity reached a certain value, the pole-climbing phenomenon would occur) during the CPAM aqueous two-phase polymerization was a key point to the dispersion polymerization in inorganic salt solution.

\section{Acknowledgements}

The authors would like to acknowledge financial supports by the National Natural Science Foundation of China (No. 20876081), Shandong Provincial Natural Science Foundation, China (No. Q2006B01) and Shandong Provincial SciTech Plan Foundation(J08LC04).

\section{References}

[1] Cho M. S., Yoon K. J., Song B. K.: Dispersion polymerization of acrylamide in aqueous solution of ammonium sulfate: Synthesis and characterization. Journal of Applied Polymer Science, 83, 1397-1405 (2002). DOI: $10.1002 / a p p .2300$

[2] McCormick C. L., Blackmon K. P., Elliott D. L.: Water-soluble copolymers. XIII. Copolymers of acrylamide with sodium-3-acrylamido-3-methylbutanoate: Solution properties. Journal of polymer Science Part A: Polymer Chemistry, 24, 2619-2634 (1986). DOI: 10.1002/pola.1986.080241019

[3] Fan A., Turro N. J., Somasundaran P.: A study of dual polymer flocculation. Colloids and Surfaces A: Physicochemical and Engineering Aspects, 162, 141148 (2000). DOI: 10.1016/S0927-7757(99)00252-6

[4] Yan R. X.: Water soluble polymer. Chemical Industry Press, Beijing (1998).

[5] Fang D. B., Guo R. W., Ha R. H.: Acrylamide polymer. Chemical Industry Press, Beijing (2006).

[6] Wu Y. M., Chen Q. F., Xu J., Bi J. M.: Aqueous dispersion polymerization of acrylamide with quaternary ammonium cationic comonomer. Journal of Applied Polymer Science, 108, 134-139 (2008).

DOI: $\underline{10.1002 / a p p .27464}$

[7] Guha S., Mandal B. M.: Dispersion polymerization of acrylamide: III. Partial isopropyl ester of poly(vinyl methyl ether-alt-maleic anhydride) as a stabilizer. Journal of Colloid and Interface Science, 271, 55-59 (2004).

DOI: $\underline{10.1016 / j . j c i s .2003 .10 .023}$

[8] Song B. K., Cho M. S., Yoon K. J., Lee D. C.: Dispersion polymerization of acrylamide with quaternary ammonium cationic comonomer in aqueous solution. Journal of Applied Polymer Science, 87, 1101-1108 (2003).

DOI: 10.1002/app.11559

[9] Ray B., Mandal B. M.: Dispersion polymerization of acrylamide: Part II. 2,2'-azobisisobutyronitrile initiator. Journal of Polymer Science Part A: Polymer Chemistry, 37, 493-499 (1999).

DOI: 10.1002/(SICI)1099-0518(19990215)37:4<493:: AID-POLA13>3.0.CO;2-Y 
[10] Chen D., Liu X., Yue Y., Zhang W., Wang P.: Dispersion copolymerization of acrylamide with quaternary ammonium cationic monomer in aqueous salts solution. European Polymer Journal, 42, 1284-1297 (2006).

DOI: $\underline{10.1016 / j . e u r p o l y m j .2005 .12 .007}$

[11] Ye Q., Zhang Z., Ge X.: Highly efficient flocculant synthesized through the dispersion copolymerization of water-soluble monomers induced by $\gamma$-ray irradiation: Synthesis and polymerization kinetics. Journal of Applied Polymer Science, 89, 2108-2115 (2003). DOI: 10.1002/app.12356

[12] Yankov D. S., Trusler J. P. M., Yordanov B. Y., Stateva R. P.: Influence of lactic acid on the formation of aqueous two-phase systems containing poly(ethylene glycol) and phosphates. Journal of Chemical and Engineering Data, 53, 1309-1315 (2008).

DOI: $10.1021 / \mathrm{je} 800016 \mathrm{r}$

[13] Zhang Y., Trabbic-Carlson K., Albertorio F., Chilkoti A., Cremer P. S.: Aqueous two-phase system formation kinetics for elastin-like polypeptides of varying chain length. Biomacromolecules, 7, 2192-2199 (2006).

DOI: $\underline{10.1021 / \mathrm{bm} 060254 \mathrm{y}}$

[14] Hatti-Kaul R.: Aqueous two-phase systems: A general overview. Molecular Biotechnology, 19, 269-272 (2001).

DOI: $10.1385 / \mathrm{MB}: 19: 3: 269$
[15] Schindler J., Lewandrowski U., Sickmann A., Friauf E.: Aqueous polymer two-phase syetem for the proteomic analysis of plasma membranes from minute brain samples. Journal of Proteome Research, 7, 432442 (2008).

DOI: $10.1021 / \mathrm{pr} 0704736$

[16] Kawaguchi S., Ito K.: Dispersion polymerization. Advances in Polymer Science, 175, 299-328 (2005).

DOI: $10.1007 / \mathrm{b} 14102$

[17] Shan G. R., Cao Z. H.: A new polymerization method and kinetics for acrylamide: Aqueous two-phase polymerization. Journal of Applied Polymer Science, 111, 1409-1416 (2009).

DOI: $10.1002 /$ app.29167

[18] Xu J., Wu Y., Wang C., Wang Y.: Dispersion polymerization of acrylamide with 2-acrylamide-2-methyl1-propane sulfonate in aqueous solution of sodium sulfate. Journal of Polymer Research, 16, 569-575 (2009).

DOI: $10.1007 / \mathrm{s} 10965-008-9261-8$

[19] Colombani D.: Chain-growth control in free radical polymerization. Progress in Polymer Science, 22, 1649-1720 (1997). DOI: $\underline{10.1016 / \mathrm{S} 0079-6700(97) 00022-1}$ 